\title{
Sigmoid Volvulus in the Paediatric Population
}

MV Vincent ${ }^{1}$, L Kandamaran ${ }^{2}$

\begin{abstract}
Sigmoid volvulus is a relatively rare condition commonly associated with the elderly. Because of this strong link with elderly adults, children- including neonates, who are by no means immune to the condition, are more prone to be misdiagnosed and suffer the consequences of a delay in diagnosis.
\end{abstract}

Keywords: Children, chronic constipation, hirschsprung's disease, sigmoid volvulus

From: ${ }^{1}$ Department of Surgery, The Queen Elizabeth Hospital, Barbados, ${ }^{2}$ Department of Radiology, The Queen Elizabeth Hospital, Barbados.

Correspondence: Dr M Vincent, Department of Surgery, The Queen Elizabeth Hospital Martindales Road, St Michael, Barbados. Fax: 1-246-429 5374, e-mail: michvincent@yahoo.com 


\section{INTRODUCTION}

Sigmoid volvulus is an extremely rare condition in the paediatric population. Because it is commonly associated with the debilitated elderly, and can have a chronic presentation with episodes of spontaneous resolution, both the presentation and diagnosis are usually delayed in children. As such the condition is associated with a higher incidence of bowel gangrene in the pediatric population, with an associated overall mortality of $6 \%$.

\section{CASE REPORT}

A 10-year old boy who was mentally challenged presented to the Accident and Emergency department with a one day history of vomiting associated with abdominal pain and distension. On presentation parents gave a history of chronic constipation over the past 8 years, which had been treated with dietary modification, laxatives and regular enemas. This however had never brought complete relief and he had continued to experience constipation marked by the passage of multiple small volume stools 3 to 4 times per day with intermittent episodes of abdominal distension. This had continued until two days prior to presentation when his stools had become watery.

On examination he was noted to be ill-looking and moderately dehydrated. He was tachycardic with a pulse rate of 130 per minute and tachypneic at 68 breaths per minute. His abdomen was globally distended, tympanitic and tense but with no evidence of peritonism. A digital rectal examination revealed a capacious rectum loaded with soft brown stool.

An abdominal xray was performed which showed a markedly distended loop of large bowel with a classic coffee bean sign (Fig 1) Once the child was resuscitated and stabilized a CT scan of the abdomen and pelvis was performed which revealed gross distension of the sigmoid colon - to a maximal diameter of $10 \mathrm{~cm}$ with associated narrowing of its proximal 
segment, rectal distension and collapsed small bowel. The presence of whorls correlating to a twisted mesentery from a volved segment of bowel was carefully sought but not noted. There was no evidence of pneumoperitoneum. In addition severe bilateral hydroureteronephrosis was also noted, more marked on the left side (Grade 4). (Fig 2)

Based on the child's longstanding history of chronic constipation with associated intermittent abdominal distention, recent onset of the passage of watery stools, hemodynamic instability on presentation and the radiological findings of severe bilateral hydroureteronephrosis- suggesting a long-standing pathology, a diagnosis of Hirschsprung's enterocolitis was made. He was admitted to the pediatric intensive care unit where resuscitation was continued, broad spectrum antibiotics started (Cefazolin and Metronidazole), and a nasogastric tube passed. A rectal washout was then performed which led to a decrease in abdominal distension after output of a large amount of gas and pasty brown stool.

On the following morning however, 18 hours after admission a repeat rectal washout failed to achieve any decrease in abdominal distension and the child's vital signs which had previously started to normalize bagan to deteriorate. In addition he gradually developed worsening respiratory distress requiring intubation and increased circulatory support necessitating inotropic support. A diagnosis of Sigmoid Volvulus with compromised bowel was then entertained and he was taken to theatre 30 hours after admission for exploratory laparotomy.

The abdomen was opened via a Pfannenstiel incision and a sigmoid volvulus was noted (with a 360 degree twist) with most of the sigmoid itself being gangrenous. The bowel proximally was collapsed but the rectum was noted to be markedly distended. Without untwisting the volved gangrenous sigmoid, sigmoidectomy was performed with formation of a distal descending colostomy and a Hartmann's procedure. Postoperatively the child 
gradually recovered spending four days in the pediatric intensive care unit where he was gradually weaned off inotropic support and eventually extubated on the $5^{\text {th }}$ postoperative day. He was then discharged to the general pediatric surgical ward where nasogastric tube feeds were commenced in the presence of a healthy functioning stoma. The child was discharged home on the $13^{\text {th }}$ postoperative day on a normal diet.

Six weeks after discharge he was readmitted electively as a day case for open rectal biopsy, which confirmed the presence of ganglion cells, thus excluding the diagnosis of associated Hirschsprung's disease with sigmoid volvulus. He subsequently underwent reversal of Hartman's and remains completely well with no recurrent episodes of large bowel volvulus at 6 months follow up.

\section{DISCUSSION}

Sigmoid volvulus (SV) occurs when a redundant sigmoid colon twist around its narrowbased, unusually long mesentery. This twist can vary from 90 degrees to 360 degrees in either a clockwise or counter-clockwise direction. Although classically a disease of the debilitated elderly, it occasionally presents in children -including neonates (1-5), either in isolation or with predisposing conditions such as intestinal malrotation, anal stenosis, imperforate anus, congenital bands, mental retardation and more commonly chronic constipation and Hirschsprung's disease (HD) (1-3, 6 -12).

There are two distinct presentations- acute and chronic $(9,13)$. In the acute presentation the duration of symptoms is typically less than 36 hours, associated with severe abdominal pain and distension, early gangrene and circulatory collapse. In chronic cases - as in this index case- there is the history of chronic intermittent episodes of abdominal pain that have been present for months to years. During this time, bouts of abdominal pain are 
frequently relieved by the passage of flatus and/or stool, which may well coincide with spontaneous reduction of the volved sigmoid $(9,13)$. It is particularly in this subset of patients that the diagnosis is continually missed.

The mean age of presentation in children is 7 to $12 \mathrm{yrs}(4,8,9,14)$, with most series demonstrating a male preponderance $(4,7,9,12,14)$. The classic presentation is that of vomiting, abdominal pain and constipation $(3,7,8,9,15)$. An interesting observation is the absence of any abdominal tenderness on clinical examination in most children with sigmoid volvulus $(3,9)$.

The diagnosis can be confirmed when a plain abdominal xray shows a hugely dilated sigmoid colon with the classic coffee bean sign- a dilated sigmoid loop arising from the pelvis into the upper part of the abdomen (16), suggesting a closed loop obstruction- as was apparent in this case (Fig 1). In addition a single air-fluid level as opposed to multiple airfluid levels is even more in keeping with the diagnosis (14). However it must be noted that over two thirds of children with sigmoid volvulus will not have the classic coffee bean sign $(6,9,14)$. Barium enema studies have also been employed as a diagnostic tool; the diagnosis being confirmed in the presence of the bird beak sign, which is formed as the upper rectum is twisted closed $(9,10,13,16)$. If a CT scan or MRI is performed the classic whorls correlating with the twist in the mesentery is noted (6), though this was not present in our case. Nonetheless a plain abdominal xray alone, showing colonic distension together with history and clinical examination should be enough to suspect and/or establish the diagnosis (13).

While the majority of cases are treated surgically, non-surgical methods are increasingly being used with great success in children who are stable with no evidence of hypovolaemic or septic shock and no pneumoperitoneum $(6,8,9,17)$. Reduction by barium enema has been successfully employed in about three quarters of pediatric patients in 
reported series (9). Others have successfully employed use of endoscopic/colonoscopic reduction with success rates of over $95 \%(8,10)$.

However, these nonsurgical approaches are temporizing measures only, associated with an extremely high rate of recurrence, with most children eventually requiring sigmoidectomy $(8,13,14,17)$. The noted exception to this dogma is in neonatal sigmoid volvulus where barium or water soluble contrast enema is permanently therapeutic, with recurrence being exceptional on long term follow up $(2,3)$.

In summary, though sigmoid volvulus is classically a disease of the elderly, it needs to be suspected in children particularly when radiologic investigations reveal the presence of dilated colon, on the background of constipation, abdominal pain, abdominal distension, and vomiting $(8,9)$. With increased awareness of its possibility in children and neonates, it is hoped that delays in diagnosis (as occurred in this case) will be markedly reduced leading to earlier presentations, a decreased incidence of gangrenous bowel and lower mortality rates when compared to adults.

\section{REFERENCES}

1. Janik JS, Humphrey R, Nagari HS. Sigmoid volvulus in a neonate with imperforate anus. J Pediatr Surg 1983; 18: 636-8.

2. Pastore V, Basile A, Cocomazzi R, Pastore M, Bartoli F. Sigmoid volvulus in a neonate: Case report and review of literature. Afr J Paediatr Surg 2013; 10: 390-2.

3. De Caluwé D, Kelleher J, Corbally MT. Neonatal sigmoid volvulus: A complication of anal stenosis. J Pediatr Surg 2001; 36:1079-81. 
4. Puneet, Khanna R, Gangopadhyay AN, Shahoo SP, Khanna AK. Sigmoid volvulus in childhood: report of six cases. Pediatr Surg Int 2000; 16: 132-3.

5. Weeratunga ND, Samarakoon LB, Lamahewage AK. Sigmoid Volvulus Causing Intestinal Obstruction in A Child - A Case Report and Review of Literature. J Pediatr Neonatal Care 2015; 2: 00067. DOI: 10.15406/jpnc.2015.02.00067.

6. Zeng M, Amodio J, Schwarz S, Garrow E, Xu J, Rabinowitz SS. Hirschsprung's disease presenting as sigmoid volvulus: report and review of the literature. J Pediatr Surg 2013; 48: 243-6.

7. Atamanalp SS, Yildirgan MI, Basoglu M, Kantarci M, Yilmaz I. Sigmoid colon volvulus in children: review of 19 cases. Pediatr Surg Int 2004; 20:492-5.

8. Colinet S, Rebeuh J, Gottrand F, Kalach N, Paquot I, Djeddi D, Le Henaff G, Rebouissoux L, Robert V et al. Presentation and endoscopic management of sigmoid volvulus in children. Eur J Pediatr 2015; 174: 965-9.

9. Salas S, Angel CA, Salas N, Murillo C, Swischuk L. Sogmoid volvulus in children and adolescents. J Am Coll Surg. 2000; 190: 717-23.

10. Albert A, Nolan TL, Weidner BC. Sigmoid volvulus in 16 year old boy with an associated anomalous congenital band. Am Surg 2013; 79: 1140-1.

11. Choudhury CR, Ghosh D, Biswas S. Sigmoid volvulus in a young patient: beware of Hirschsprung's disease. Saudi Surg J 2013; 1: 29-31.

12. Sarioglu A, Tanyel FC, Buyukpamukcu N, Hicsonmez A. Colonic volvulus: a rare presentation of Hirschsprung's disease. J Pediatr Surg 1997; 32:117-8.

13. Ismail A. Recurrent colonic volvulus in children. J Pediatr Surg 1997; 32:1739-42.

14. Smith SD, Golladay ES, Wagner C, Seibert JJ. Sigmoid volvulus in children. South Med J. 1990; 83: 778-81. 
15. Samuel M, Boddy SA, Nicholls E, Capps S. Large bowel volvulus in childhood. Aust NZL Surg 2000; 70: 258-262.

16. Baden LR. Beans and beaks. N Engl J Med 2013; 369: 25.

17. Ton MN, Ruzal-Shapiro C, Stolar C, Kazlow PG. Recurrent sigmoid volvulus in a sixteen-year-old boy: case report and review of the literature. J Pediatr Surg 2004; 39:1434-6. 


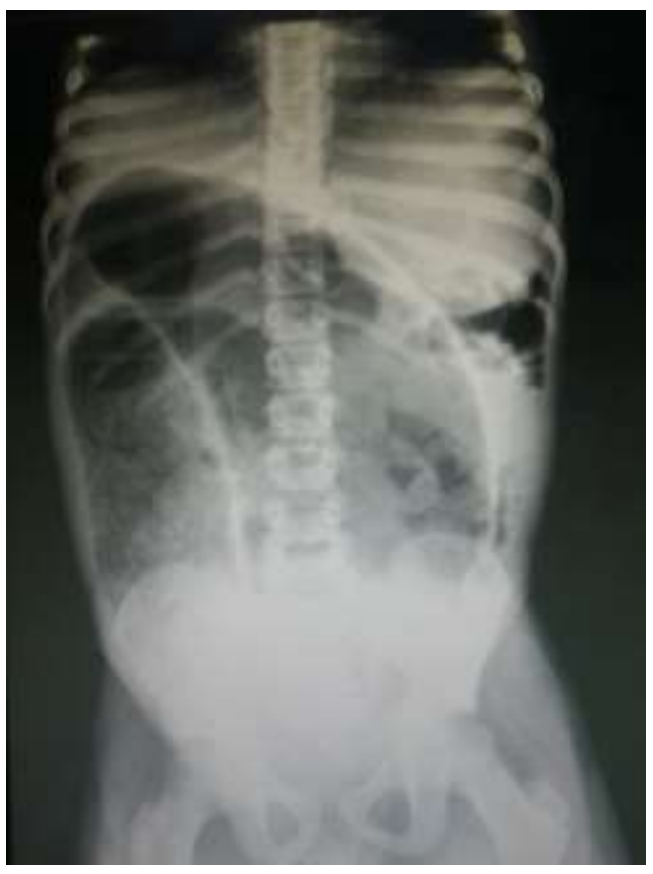

Fig. 1: Plain abdominal xray showing a hugely dilated sigmoid colon (the classic coffee bean sign).

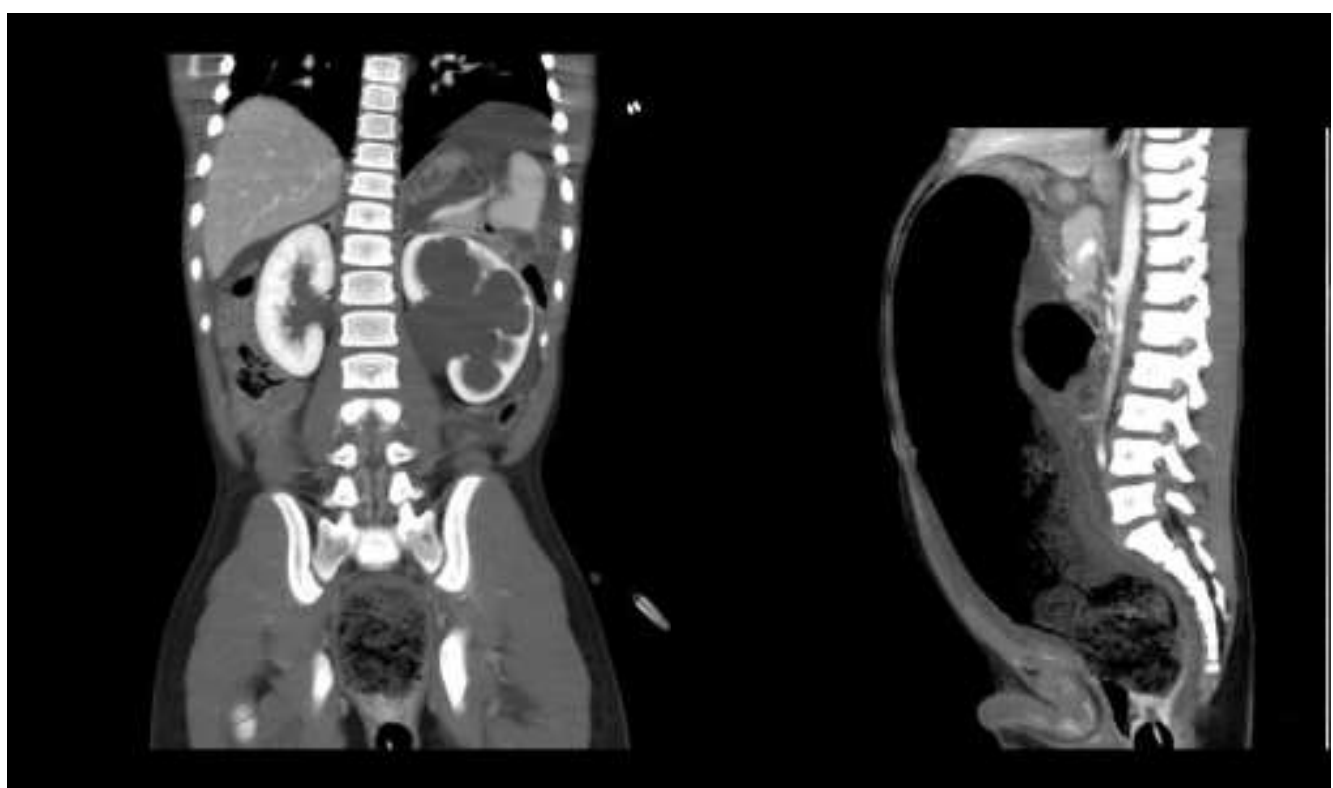

Fig. 2: Abdominal CT scan image showing gross distension of the sigmoid colon with associated narrowing of its proximal segment, rectal distension, collapsed small bowel and bilateral hydroureteronephrosis. 